\title{
Manejo da dor durante o parto: Um enfoque nas medidas não farmacológicas
}

\author{
Pain management during childbirth: A focus on non-pharmacological measures \\ Manejo del dolor durante el parto: Un enfoque en medidas no farmacológicas
}

Recebido: 10/04/2021 | Revisado: 16/04/2021 | Aceito: 30/04/2021 | Publicado: 14/05/2021

Ana Roza Carvalho Silva

ORCID: https://orcid.org/0000-0002-8804-2832 Christus Faculdade do Piauí, Brasil

E-mail: anaroza.carvalho@gmail.com

Sabrina Beatriz Mendes Nery

ORCID: https://orcid.org/0000-0002-8254-0152

Christus Faculdade do Piauí, Brasil

E-mail:sabrinaanery2019@gmail.com

Guilherme Antônio Lopes de Oliveira

ORCID: https://orcid.org/0000-0003-3820-0502

Christus Faculdade do Piauí, Brasil

E-mail: guilhermelopes@live.com

Evaldo Sales Leal

ORCID: https://orcid.org/0000-0002-1424-9048 Christus Faculdade do Piauí, Brasil

E-mail: evaldosleal@bol.com

Mauro Gustavo Amaral Brito

ORCID: https://orcid.org/0000-0002-9551-4025

Christus Faculdade do Piauí, Brasil

E-mail: drmaurogustavo@hotmail.com

Neirigelson Ferreira de Barros Leite

ORCID: https://orcid.org/0000-0001-6505-1368

Christus Faculdade do Piauí, Brasil

E-mail: neirigelson@hotmail.com

Gabriel Mauriz de Moura Rocha

ORCID: https://orcid.org/0000-0003-1454-0414

Christus Faculdade do Piauí, Brasil

E-mail: mauriz45@hotmail.com

Iani Clara Oliveira do Nascimento

ORCID: https://orcid.org/0000-0002-2131-352X

Christus Faculdade do Piauí, Brasil

E-mail: Ianioliveira46@gmail.com

Anne Beatriz do Nascimento Souza

ORCID: https://orcid.org/0000-0002-3113-0866

Christus Faculdade do Piauí, Brasil

E-mail: annebea0498@gmail.com

Laysa Cristine Medeiros de Carvalho

ORCID: https://orcid.org/0000-0003-4435-4882

Christus Faculdade do Piauí, Brasil

E-mail: laysacristine18@hotmail.com

Laisa Garcia Matos

ORCID: https://orcid.org/0000-0002-7137-5585

Christus Faculdade do Piauí, Brasil

E-mail: laisagarciamatos45@gmail.com

Josyskleydon Fellipe da Silva Vieira

ORCID: https://orcid.org/0000-0003-1989-5193

Christus Faculdade do Piauí, Brasil

E-mail: OliveiraJosyskleydonfellipe2703@gmail.com

Ana Giselle Lima da Silva

ORCID: https://orcid.org/0000-0003-1274-603X

Christus Faculdade do Piauí, Brasil

E-mail: anagisellelimadasilva@gmail.com

Antonia Jessica da Silva

ORCID: https://orcid.org/0000-0001-6201-9696

Christus Faculdade do Piauí, Brasil

E-mail: Jessica.silva622@hotmail.com

Carliane Maria de Araújo Souza

ORCID: https://orcid.org/0000-0002-8196-0008

Christus Faculdade do Piauí, Brasil

E-mail: kku_ka@hotmail.com 


\title{
Resumo
}

O parto é considerado um evento complexo, capaz de ser positivamente marcante no âmbito emocional da gestante ou até mesmo doloroso e traumático. Oferecer boas práticas à mulher é necessário e significa dá suporte seguro e de qualidade, levando em consideração o conforto físico e emocional, respeitando e auxiliando na forma como ela deseja parir e principalmente oferecendo-a técnicas capazes de aliviar suas dores. Esse estudo visou avaliar a eficácia dos métodos não farmacológicos para alívio da dor durante o parto e o mesmo tratou-se de um estudo observacional descritivo do tipo série de casos. Os participantes da pesquisa foram parturientes admitidas na Maternidade Dr. João Bandeira Monte e que se encaixassem em alguns critérios, sendo estes: parturientes com faixa etária a partir de 18 anos, de baixo risco, que estivessem na fase ativa do trabalho de parto, com máximo de 6 centímetros de dilatação do colo uterino e que concordassem em receber os métodos não farmacológicos, além de aceitarem participar da pesquisa sob entendimento e assinatura do Termo de Consentimento Livre e Esclarecido. Foi utilizado uma terapêutica de métodos não farmacológicos combinados e isolado, para alívio da dor das parturientes e aplicado a Escala Visual Analógica para avaliar a eficácia desses métodos. Para análise desses dados, foi utilizado tabelas e gráficos para tratamento estatístico com médias e percentuais no Microsoft Office Excel 2016. O resultado desse estudo pôde demonstrar que há eficácia dentre os métodos que foram utilizados dentro do contexto das parturientes que foram atendidas, porém, há quesitos determinantes para que esses possam funcionar de forma individual em cada mulher. Deve ser levado em consideração a subjetividade, as escolhas, o conhecimento prévio da parturiente sobre esses métodos, a interação paciente-profissional, entre outros aspectos.

Palavras-chave: Aliviamento; Dor do parto; Escala visual analógica; Humanização.

\begin{abstract}
Childbirth is considered a complex event, capable of being positively striking in the emotional sphere of the pregnant woman or even painful and traumatic. Offering good practices to women is necessary and means providing safe and quality support, taking into account physical and emotional comfort, respecting and helping in the way she wishes to give birth and, above all, offering her techniques capable of relieving her pain. This study aimed to evaluate the effectiveness of non-pharmacological methods for pain relief during childbirth and it was an observational descriptive study of the case series type. The research participants were parturients admitted to the Dr. João Bandeira Monte Maternity Hospital and who met some criteria, namely: parturients with an age range from 18 years old, of low risk, who were in the active phase of labor, with maximum of 6 centimeters of dilation of the uterine cervix and who agreed to receive non-pharmacological methods, in addition to accepting to participate in the research under the understanding and signing the Free and Informed Consent Form. Combined and isolated non-pharmacological methods were used to relieve the pain of parturients and the Visual Analogue Scale was applied to evaluate the effectiveness of these methods. For the analysis of this data, tables and graphs were used for statistical treatment with averages and percentages in Microsoft Office Excel 2016. The result of this study could demonstrate that there is effectiveness among the methods that were used within the context of the parturients who were attended, however, determinant requirements so that these can work individually in each woman. Subjectivity, choices, the parturient's previous knowledge about these methods, patient-professional interaction, among other aspects, must be taken into account.
\end{abstract}

Keywords: Relief; Labor pain; Analogic visual scale; Humanization.

\section{Resumen}

El parto se considera un evento complejo, capaz de impactar positivamente en el ámbito emocional de la embarazada o incluso doloroso y traumático. Ofrecer buenas prácticas a la mujer es necesario y significa brindar un apoyo seguro y de calidad, teniendo en cuenta el confort físico y emocional, respetando y ayudando en la forma en que desea dar a luz y, sobre todo, ofreciendo sus técnicas capaces de aliviar su dolor. Este estudio tuvo como objetivo evaluar la efectividad de métodos no farmacológicos para el alivio del dolor durante el parto y fue un estudio descriptivo observacional del tipo serie de casos. Las participantes de la investigación fueron parturientas ingresadas en la Maternidad Dr. João Bandeira Monte y que cumplieron algunos criterios, a saber: parturientas con un rango de edad de 18 años, de bajo riesgo, que se encontraban en la fase activa del parto, con un máximo de 6 centímetros de dilatación cervical y quienes accedieron a recibir métodos no farmacológicos, además de aceptar participar en la investigación bajo el entendimiento y firma del Formulario de Consentimiento Libre e Informado. Se utilizaron métodos no farmacológicos combinados y aislados para aliviar el dolor de las parturientas y se aplicó la Escala Visual Analógica para evaluar la efectividad de estos métodos. Para el análisis de estos datos se utilizaron tablas y gráficos para el tratamiento estadístico con promedios y porcentajes en Microsoft Office Excel 2016. El resultado de este estudio pudo demostrar que existe efectividad entre los métodos que se utilizaron en el contexto de las parturientas que fueron atendido, sin embargo, existen requisitos determinantes para que estos puedan trabajar individualmente en cada mujer. Se debe tener en cuenta la subjetividad, las elecciones, el conocimiento previo de la parturienta sobre estos métodos, la interacción paciente-profesional, entre otros aspectos.

Palabras clave: Alivio; El dolor del parto; Escala visual analógica; Humanización. 


\section{Introdução}

O parto é considerado um evento complexo, capaz de ser positivamente marcante no âmbito emocional da gestante ou até mesmo doloroso e traumático. O processo parturitivo pode ser caracterizado por alterações hormonais e mecânicas, que inicialmente promove contrações uterinas e dilatação do colo, resultando em dores progressivas, e findando-se na expulsão fetal. Reconhecendo que a dor pode acarretar prejuízos para mãe e bebê, é sabido atualmente que há a necessidade de aliviá-la (Gallo et al., 2011).

Historicamente, a dor provocada pelo parto normal é correlacionada com a ideia de sofrimento, gerando assim medo e insegurança à mulher, porém, ela não deve ser vista como um estado patológico e sim, como uma função biológica. Ela pode ser caracterizada como orgânica, aguda, visceral e difusa, evoluindo para somática e contínua, sendo resultado de estímulos provocados pelas contrações uterinas, podendo ser influenciada pelo estado emocional da parturiente e por fatores ambientais (Almeira, Medeiros \& Souza, 2012).

A Organização Mundial de Saúde (OMS) preconiza que a assistência ao parto deve ter o mínimo de intervenções médicas, mantendo-o mais natural possível, intervindo apenas quando necessário. Busca-se assim a garantia da segurança da mãe e do filho, tornando o processo o mais agradável possível, sem o exagero de práticas intervencionista e medicalizadas. Apesar das recomendações a incidência de parto por via cirúrgica tem aumentado em todo o mundo (Patah \& Malik, 2011).

Segundo o Sistema Nacional de Informações de Nascidos Vivos (SINASC) houve um aumento alarmante do número de cesáreas no Brasil, saindo de 32\% em 2000, para 52,3\% em 2010. As regiões Sudeste, Sul e Centro-Oeste apresentaram aumento de quase $60 \%$ entre esses anos, enquanto que as regiões norte e nordeste obtiveram maiores percentuais de $52 \%$ e $73,2 \%$, respectivamente (Brasil, 2012). A escolha por essa via de parto tem forte influência dos sentimentos e experiências traumáticas vivenciadas por mulheres em partos normais e uso de intervenções agressivas e desnecessárias, muitas vezes aliadas a falta de suporte emocional e humanização na assistência (Oliveira \& Cruz, 2014).

A prática da humanização no processo parturitivo, favorece mudanças no atendimento à gestante, oferecendo a ela assistência as suas necessidades e garantindo o uso de práticas seguras que incluam o respeito ao processo fisiológico. Com o intuito de concentrar esforços para reduzir as taxas de morbimortalidade materna e fetal, o Ministério da Saúde (MS) instituiu o Programa de Humanização ao Pré-natal e Nascimento (PHPN) para que a mulher e seu bebê tenham um atendimento digno e de qualidade de forma humanizada e segura (Santos \& Araujo, 2016).

Objetivando fortalecer o PHPN, o MS criou a Rede Cegonha, regulamentada por meio da portaria $\mathrm{n}^{\circ} 1.459$, de 24 de junho de 2011, que visa garantir atenção humanizada ao parto e puerpério, além do direito à criança ao nascer seguro. Preconiza também, a adoção de boas práticas, como medidas, cuidados e atividades que objetivam favorecer o parto seguro, oferecendo a mulher empoderamento e protagonismo nesse evento (Medeiros et al., 2015). Com base no exposto, cabe ressaltar que a maternidade João Bandeira Monte localizada no município de Piripiri se tornou referência na realização de parto humanizado no território dos cocais por participar e seguir as instruções da Rede Cegonha.

Segundo Medeiros et al. (2015) oferecer boas práticas à mulher, significa dá suporte seguro e de qualidade, levando em consideração o conforto físico e emocional, respeitando e auxiliando na forma como ela deseja parir e principalmente oferecendo-a técnicas capazes de aliviar suas dores. Portanto é imprescindível que se tenha conhecimento adquirido através de estudos sobre a eficácia dos métodos que estão sendo aplicados dentro das unidades hospitalares.

Tendo conhecimento da eficácia desses métodos é possível proporcionar benefícios não só para a gestante, mas também para a instituição oferecedora da assistência, pois diminuem a necessidade intervencionista de medicações, instrumentais e procedimentos invasivos. Além de abater as chances de infecções, hemorragias e eventos trombóticos no pósparto. Contudo, com o emprego desses métodos é possível oferecer tranquilidade, conforto e alívio para a gestante, 
configurando primor na assistência ao processo de parturição e além disso, ressaltar a sua influência na otimização dos gastos da instituição.

Os principais meios utilizados para o alívio da dor dentro do processo de parturição são as técnicas não farmacológicas, que podem reforçar a autonomia da mulher, efetivando sua participação ativa durante o trabalho de parto e parto. Dentre esses métodos pode-se mencionar o suporte contínuo, massoterapia, mobilidade materna, deambulação, banho de chuveiro e de imersão, exercícios respiratórios, entre outros (Gallo et al., 2011).

A dor deve ser manejada de forma individual, levando em consideração as particularidades de cada mulher, porém, com a sua subjetividade, torna-se dificultosa estimar seu limiar. No entanto existe uma escala que pode ser usada para sua aferição, sendo esta, a escala visual analógica (EVA), que auxilia na mensuração da intensidade da dor, por meio de uma linha horizontal ou vertical que contém classificações (leve, moderada e intensa), nas quais o paciente vai assinalar, facilitando assim a compreensão do profissional quanto a intensidade de dor referida (Barbieir et al., 2013).

O presente estudo visou avaliar a eficácia dos métodos não farmacológicos para alívio da dor durante o parto, ressaltando a importância do processo de humanização dentro do contexto parturitivo, além de detalhar as facetas da dor, bem como aplicar a escala visual analógica para analisá-la.

\subsection{Caracterizando a dor do parto}

A dor do parto é uma característica peculiar ao evento parturitivo e historicamente é associada a ideia de sofrimento. Culturalmente tem contribuído para que o parto normal tenha significado de evento traumático para a mulher. Essa ideia de sofrimento tem influenciado no aumento da solicitação de "cesárea a pedido" e consequentemente na elevação dos seus índices, ultrapassando a taxa anual aceito pela OMS de 15\%, passando assim a construir um problema social e de saúde pública, pois aumenta a chance de eventos adversos para mãe e para o bebê (Almeida, Medeiros \& Souza, 2012).

Com a progressão do trabalho de parto, é comum que se inicie sensações dolorosas, provenientes das contrações que estão presentes tanto no primeiro, como no segundo estágio do TP e da dilatação do colo uterino. A dor sentida pela mulher pode sofrer influência de diversos fatores, como os socioculturais, psicológicos, individuais e do ambiente em que é atendida, por isso deve ser respeitada e não deve ser subestimada por quem presta a assistência, devendo assim atendê-la de forma holística, humanizada e prover cuidados, com o intuito de amenizar as dores e desconfortos, para melhor experiência da mulher e progressão do parto.

Para Brandolfi et al. (2017) o primeiro estágio é aquele que compreende o período de dilatação do colo uterino, quando ele apaga por completo ocorre o processo de descida e à medida que o feto desce pelo canal de parto vai aumentando a sensação dolorosa, bem como o estresse emocional, o medo e a ansiedade. Durante ele, se pode considerar a dor como visceral, não localizada, intermitente e difusa, diferente do próximo estágio, que é de expulsão fetal, onde a dor tem característica somática, intensa, contínua e localizada. A sensação dolorosa sentida neste momento é individual e multifatorial, contando ainda com a influência da lesão tecidual.

Um instrumento capaz de avaliar a percepção e sensação dolorosa proveniente da dor de parto é a EVA, que consiste em uma linha horizontal ou vertical de 10 centímetros, na qual suas extremidades vai de 0 a 10 . Ela pode ser dividida em três classificações, sendo de 0 a 2, dor leve, de 3 a 7, dor moderada e 8 a 10, dor intensa. Serve como ferramenta para melhor quantificar a dor que o cliente está sentindo e repensar se a terapêutica utilizada está sendo eficaz para o tratamento dela (Castro, Castro \& Mendonça, 2012).

O alívio da dor não está necessariamente ligada a uma experiência de parto mais aceitável. No entanto, se a parturiente se sentir acolhida e confortada, essa experiência pode tornar menos traumática. As gestantes não temem apenas a 
dor, mas receiam as práticas e intervenções que irão receber durante o cuidado, dentre eles o atendimento impessoal, distante e frio por parte daqueles que estão envolvidos no processo, prestando esse cuidado (Almeida, Acosta \& Pinhal, 2015).

\subsection{Métodos não farmacológicos para alívio da dor no parto}

De acordo com as recomendações contidas no manual Maternidade Segura, existem aquelas que são comprovadamente benéficas durante o trabalho de parto e parto e que devem ser estimuladas, podendo ser citadas, a oferta de líquidos, apoio profissional, direito ao acompanhante, estimulo a posições não supinas, liberdade de posição e movimento, uso de partograma, contato pele a pele entre mãe e bebê, utilização dos métodos não farmacológicos para alívio da dor, entre outros (Organização Mundial da Saúde, 1996).

O uso dos MNFDP é proposto no intuito de substituir o uso de anestésicos, analgésicos e utilização de tecnologias invasivas durante o processo de parturição, buscando retoma-lo como evento fisiológico. Seu uso traz como benefícios, menos efeitos colaterais para a mãe e bebê, pois não se utilizam de medicações para efetiva-las e são capazes de promover sensação de controle e empoderamento da mulher (Ritter, 2012).

Para melhor entendimento serão apresentados de forma sucinta os MNFDP mais comumente utilizados, com suas características e mecanismos para amenizar a dor durante o evento parturitivo.

Quadro 1 - Métodos não farmacológicos mais utilizados e suas especificidades.

\begin{tabular}{|c|c|}
\hline Banho de chuveiro ou imersão & $\begin{array}{l}\text { Durante o banho é possível provocar vasodilatação periférica e aumento da } \\
\text { redistribuição do fluxo sanguíneo que auxiliará provocando relaxamento muscular, e } \\
\text { podendo assim agir diminuindo a dor durante as contrações. (Katzer, 2016). }\end{array}$ \\
\hline Bola suíça & $\begin{array}{l}\text { A posição vertical e a movimentação em cima da bola permitem que a força da } \\
\text { gravidade associado com o alinhamento do eixo fetal com a bacia materna, bem como a } \\
\text { abertura da pelve favoreçam a descida e a progressão fetal no canal de parto. Nesse } \\
\text { contexto a movimentação feita juntamente com a movimentação materna pode auxiliar na } \\
\text { diminuição da dor, favorecer a circulação materno-fetal, aumentar a intensidade das } \\
\text { contrações e consequentemente diminuir a duração do trabalho de parto, entre outros } \\
\text { (Barbieir et al. 2013). }\end{array}$ \\
\hline $\begin{array}{l}\text { Deambulação/ Mudança de } \\
\text { posição }\end{array}$ & $\begin{array}{l}\text { Segundo Silva et al. (2013) a deambulação é uma técnica que permite a } \\
\text { movimentação livre da mulher, não restringindo-a ao leito. Ela visa a redução do trabalho } \\
\text { de parto por meio da mobilidade pélvica que ajuda a acelerar a dilatação do colo uterino } \\
\text { como também a descida fetal, além de promover maior tolerância a dor durante o trabalho } \\
\text { de parto. }\end{array}$ \\
\hline Suporte contínuo & $\begin{array}{l}\text { O suporte contínuo é realizado como forma de dar suporte emocional e conforto } \\
\text { físico à mulher, encorajando-a durante todo o processo de parturição. Ele tem como } \\
\text { benefício ainda, a interação entre profissionais e parturiente, facilitando assim a } \\
\text { comunicação e visando garantir que as informações cheguem até a parturiente de forma } \\
\text { clara, bem como, o cumprimento de uma assistência humanizada e eficaz (Gallo et al. } \\
\text { 2011). }\end{array}$ \\
\hline Massagem & $\begin{array}{l}\text { De acordo com Araújo et al. (2018) a massagem é caracterizada como um } \\
\text { método de manipulação dos tecidos que favorece a estimulação sensorial e que tem o } \\
\text { potencial de promover alívio de dores, estimular o relaxamento, aumentar o fluxo } \\
\text { sanguíneo e a oxigenação para os tecidos, além de promover contato físico com a paciente. }\end{array}$ \\
\hline Musicoterapia & $\begin{array}{l}\text { A musicoterapia tem a capacidade de diminuir o estresse, ansiedade, aflição e } \\
\text { medo da mulher relacionado ao trabalho de parto. A redução da ansiedade, auxilia na } \\
\text { redução da pulsação cardíaca, dos esforços respiratórios e promove relaxamento, além de } \\
\text { diminuir a dor, pois tem a capacidade de tirar o foco do cérebro da sensação dolorosa. } \\
\text { (Araujo } \text { et al. 2018). }\end{array}$ \\
\hline
\end{tabular}




\begin{tabular}{|c|c|}
\hline $\begin{array}{c}\text { Elas têm como objetivo reduzir a tensão muscular e a ansiedade, corroborando } \\
\text { respiração }\end{array}$ & $\begin{array}{c}\text { para tranquilização da mente, sensação de controle da dor e relaxamento dos músculos, que } \\
\text { contribuem assim para menor consumo de oxigênio e diminuição as frequências cardíacas } \\
\text { e respiratórias (Gallo et al. } 2011) .\end{array}$ \\
As técnicas de relaxamento associada com as de respiração se mostram atrativas \\
por serem simples e por garantirem a participação ativa da mulher e controle da dor \\
durante a parturição (Silva et al. 2013).
\end{tabular}

Fonte: Pesquisador responsável (2019).

\section{Metodologia}

O presente estudo tratou-se de um estudo observacional descritivo do tipo série de casos. Um estudo descritivo como o próprio nome já sugere, vai descrever as características de uma amostra, não tendo obrigatoriedade em estabelecer relação entre elas. Já nos estudos observacionais cabe ressaltar que não há manipulação das intervenções que são realizadas diretamente nos pacientes durante o estudo, portanto o pesquisador fica restrito a observar as características, dados e informações da amostra, mas não interfere (Oliveira, Velarde \& De As, 2015).

Destaca-se que todo protocolo de realização da pesquisa foi aprovado pelo comitê de ética em pesquisa com seres humanos com número 3.588.336.

A pesquisa foi realizada no município de Piripiri, no Estado do Piauí, estando localizado no território dos cocais, a $166 \mathrm{~km}$ de distância da capital Teresina ao norte da mesma. Segundo o censo de 2010, possui uma população de 61.834 pessoas (IBGE, 2018).

O município conta com o Hospital Regional Chagas Rodrigues (HRCR), que é referência na região dos cocais, comporta setores de urgência e emergência, clínica médica, centro cirúrgico, pediatria, Unidade de Terapia Intensiva e conta ainda com a Maternidade Dr. João Bandeira Monte, referência na assistência ao parto humanizado, local esse escolhido para cenário do estudo. Tendo como critérios de inclusão, parturientes com faixa etária a partir de 18 anos, de baixo risco, que estivessem na fase ativa do trabalho de parto, com máximo de 6 centímetros de dilatação do colo uterino e que concordaram em receber os métodos não farmacológicos, além de terem aceitado participar da pesquisa sob entendimento e assinatura do Termo de Consentimento Livre e Esclarecido (TCLE).

Ficaram exclusas da pesquisa gestantes que estavam fazendo uso de analgésicos, que se negaram a participar da pesquisa e de receber a aplicação dos métodos não farmacológicos para alívio da dor, aquelas com indicação de cesariana eletiva, com cesariana anterior, ou que apresentaram qualquer patologia obstétrica, bem como aquelas que tiveram sua primeira gestação evoluída para o aborto.

Foi utilizado um instrumento para obter-se dados epidemiológicos das participantes, bem como dados gestacionais (período gestacional, gestações anteriores, pré-natal, intercorrências, doenças associadas), estes puderam ser colhidos por relatos das pacientes, por informações contidas no prontuário e/ou partograma. Foi utilizada a Escala Visual Analógica (inserida no instrumento) para mensurar a dor antes e depois da aplicação dos métodos não farmacológicos para alívio da dor nas parturientes. Na qual possuía uma escala composta por uma linha horizontal que vai de 0 a 10 , no qual 0 a 2 é considerado leve, 3 a 7 moderada e 8 a 10 intensa.

Os participantes foram submetidos a riscos físicos dor e desconforto durante a entrevista e riscos psicológicos como, por exemplo, modificações nas emoções e estresse decorrente da presença do pesquisador. Com isso, foi explicado todo o procedimento para as participantes selecionadas, e que estas teriam todo o amparo por parte do pesquisador.

As informações colhidas durante a entrevista tiveram sua privacidade preservada pelo pesquisador responsável. O público alvo da pesquisa não foi identificado em nenhuma ocasião. 
Ainda conforme o item V da resolução 466/12 do CNS, os riscos precisam ser minimizados, independente do tamanho do risco. Para garantir a segurança do profissional participante da pesquisa e minimizar os possíveis riscos ocasionados por ela, as entrevistas aconteceram em ambiente confortável e de forma individualizada, diminuindo a invasão de privacidade e a quebra de confidencialidade. Todo o procedimento foi explicado para os participantes, e estes tiveram todo o amparo assistencial por parte dos pesquisadores de forma imediata e integrada, não havendo complicações durante a realização da pesquisa.

Ressalta-se que a presente pesquisa obedeceu todos os princípios éticos legais expostos na Resolução 466/12 e Resolução 580/18 do (CNS), afirmando que os participantes da pesquisa, assim como os dados coletados, não foram expostos de maneira inadequada.

Por fim, os dados foram inseridos em tabelas e gráficos para tratamento estatístico com médias e percentuais no Office Excel.

\section{Resultados e Discussão}

O estudo foi composto por uma série de três casos de mulheres em trabalho de parto. Obteve-se, primeiramente, o resultado da aplicação do instrumento com os dados epidemiológicos de cada paciente e, em seguida foi obtido o resultado da aplicação da escala visual analógica com as informações do antes e depois da aplicação dos métodos não farmacológicos para alívio da dor durante o parto.

Os métodos aplicados nas três mulheres foram realizados de forma padronizada para que se diminuísse a possibilidade de viés. Cada paciente recebeu as primeiras orientações, assinaram o TCLE e indicaram seu nível de dor na EVA, a partir daí o profissional aplicou as técnicas descritas anteriormente, iniciando pela música de preferência das parturientes e após elas foram posicionadas verticalmente na bola suíça e instituída as massagens lombossacrais durante as contrações, por fim foi aplicado a EVA novamente para avaliação pós métodos.

A EVA foi aplicada e o que foi obtido foi repassado para o instrumento. Cada paciente e seus resultados serão descritos logo abaixo:

Gráfico 1 - Nível de dor referida pelas pacientes antes e após a aplicação dos MNFDP combinados (massagem lombossacral, posicionamento materno, musicoterapia e bola suíça).

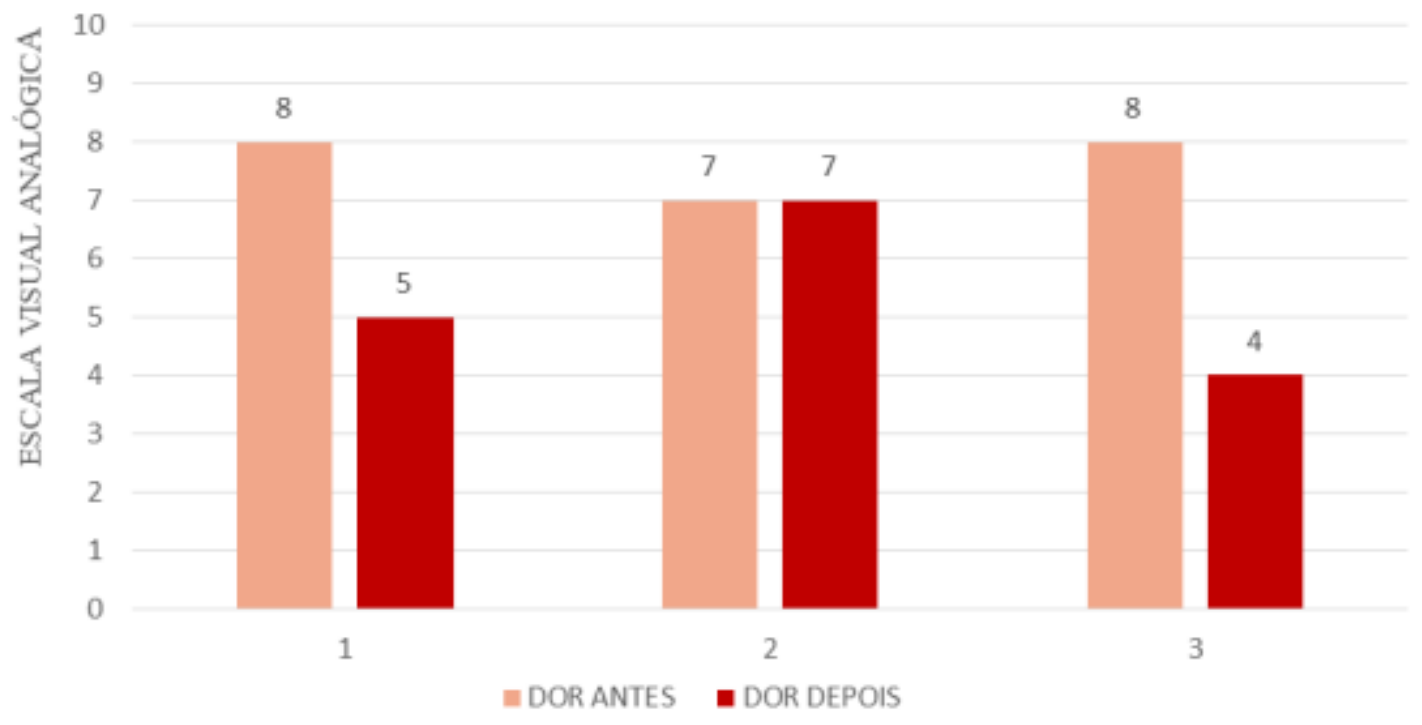

Fonte: Autores (2019). 
Gráfico 2 - Nível de dor referida pelas pacientes antes e após a aplicação do MNFDP isolado (banho de chuveiro).

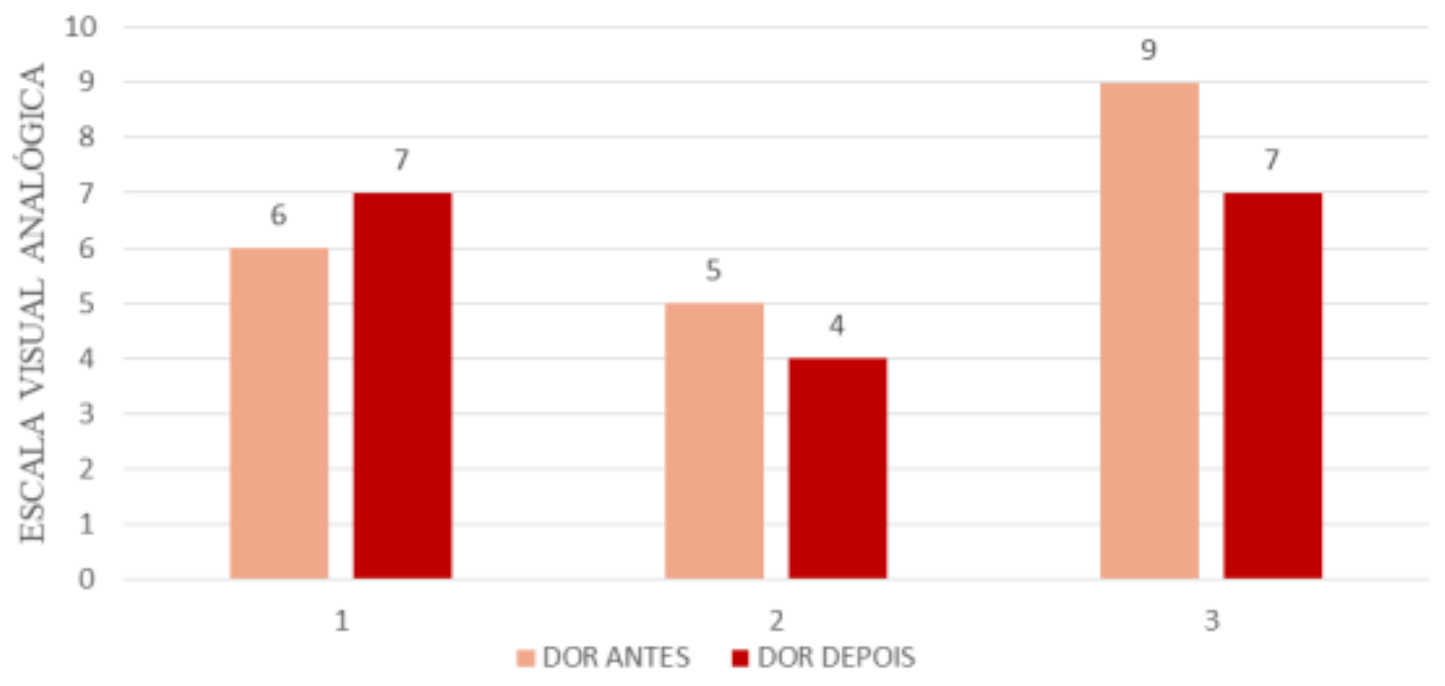

Fonte: Autores (2019).

Parturiente Número Um: 30 anos de idade, natural de Domingos Mourão, com renda familiar de até um salário mínimo, ensino médio incompleto, solteira, 2 gestações anteriores, sem doenças associadas ou intercorrências durante a gestação, se encontrava na 38 semana de gestação e participou de 8 consultas de pré-natal

A partir da interpretação da Gráfico 1 , pode-se observar que a paciente no $6^{\circ}$ centímetro de dilatação, antes da aplicação dos métodos, apresentava escore 8 na EVA o que significa que a dor era intensa, já no segundo momento que foi após a aplicação, a dor passou a ser 5, significando assim, que a dor já era moderada, pode-se concluir então, que a dor diminuiu quase $40 \%$ após a intervenção do profissional.

Interpretando o Gráfico 2 que trata-se das informações obtidas por meio da EVA analisando o método isolado, tendo assim como resultado 6 antes e 7 depois da sua aplicação. Entende-se que houve um aumento de $10 \%$ na intensidade da dor, porém continua classificada como dor moderada. Segundo Castro, Castro \& Mendonça (2012) o aumento dessa dor justifica-se pelo aumento da dilatação do colo uterino, logo o aumento é proporcional com o passar das horas de trabalho de parto. Analisando esse caso de forma particular, cabe ressaltar que não se pode afirmar que não há eficácia desse método, mas que as particularidade e subjetividades da paciente, podem ter contribuído para o não alívio da dor naquele momento.

Parturiente Número Dois: 23 anos de idade, natural de Piripiri, com renda familiar de 1 à 3 salários mínimos, ensino médio completo, casada, nenhuma gestação anterior, sem doenças associadas ou intercorrências durante a gestação, se encontrava na 39 semana de gestação e participou de 8 consultas de pré-natal.

Apresentou durante os métodos combinados escore de 7 tanto antes como depois, demostrando nenhuma alteração no limiar de dor durante os 6 centímetros. Já durante o banho de chuveiro houve uma diminuição de $10 \%$ no nível de dor, saindo de 5 para 4 que são considerados moderado. Os dados mostram que não houve diferença significativa na primeira aplicação, porém o fato de não ter aumentado, promoveu o mínimo de conforto.

Parturiente Número Três: 18 anos de idade, natural de Piripiri, com renda familiar de até um salário mínimo, ensino fundamental incompleto, em união estável, nenhuma gestação anterior, sem doenças associadas ou intercorrências durante a gestação, se encontrava na 40 semana de gestação e participou de 9 consultas de pré-natal. 
O resultado em gráficos pode descrever a dor durante os métodos combinados como intensos, pois indicava escore $8 \mathrm{e}$ passou a ser 4 moderado, caindo cerca de 50\% durante a aplicação. Durante o método isolado o escore atingiu o limiar quase máximo (9) dor intensa e diminuiu para 7 moderada, caindo cerca de $20 \%$.

Após a análise estatística dos valores gerais das 3 participantes da pesquisa, encontrou-se a média das variáveis ANTES $(7,6)$ e APÓS $(5,3)$ da aplicação dos métodos combinados, resultando numa diminuição significativa de $30 \%$ nos níveis de dor. Foi analisado estatisticamente também as médias das variáveis ANTES $(6,6)$ e DEPOIS $(6,0)$ da aplicação do método isolado, resultando numa diminuição de $9 \%$ da dor.

A dor pode sofrer diversas influências e por se tratar de algo subjetivo e pessoal, ela deve ser mensurada para permitir a escolha eficaz de algum método de alívio. A avaliação da sensação dolorosa pode ser realizada por meio de exames laboratoriais, observações comportamentais e descrições verbais e /ou escritas da dor (Nielsen, Sabatino \& Lopes, 2011). As técnicas não farmacológicas foram aplicadas e os resultados obtidos através da descrição verbal após sua aplicação, foram demostrados através de padrão numérico, porém, ela pôde ser descrita também por relato mais detalhado e observação do comportamento das pacientes.

A parturiente Número Um, por exemplo, descreveu durante a aplicação dos métodos combinados que houve um maior alívio durante esses métodos, principalmente durante a aplicação da massagem lombossacral, pois segundo ela aliviava bastante durante as contrações. Relatou ainda que o banho ajudou a relaxar, porém, não alíviou a dor. A partir de uma revisão sistemática pode se ter conhecimento de quatro estudos, do tipo ensaios clínicos e estudos qualitativos das percepções das mulheres em relação a aplicação da massagem durante o processo de trabalho de parto e o resultado desses estudos comprovou uma diminuição da dor de modo considerável tanto na primeira quanto na segunda fase do TP (Osorio et al., 2014).

A parturiente Número Dois relatou não sentir alívio durante a aplicação dos métodos combinados, porém, durante o banho ouve uma certa melhora. A paciente ficou cerca de 8 minutos embaixo do chuveiro.

Para Apolinário et al. (2016) a dor pode sofrer diversas influências e olhando sob a ótica afetiva, o medo do desconhecido, a falta de informações sobre as estratégias que são utilizadas durante o parto, pode afetar a condição psicológica e levar a produção de adrenalina e inibir os hormônios necessários para a progressão do trabalho de parto. Pode ainda acarretar resistência na hora da aplicação dos métodos e isso contribuir para o não relaxamento e consequentemente interferência no alívio da dor (Braz et al. 2014).

Em 2009 em uma maternidade pública em Natal/RN foi realizado uma pesquisa do tipo intervenção terapêutica com 30 pacientes para avaliar a eficácia dos métodos não farmacológicos, dentre eles o banho de chuveiro durante o parto e teve-se como resultado a efetividade nesse método, diminuindo assim significativamente a dor daquelas parturientes (Davim, Torres \& Dantas, 2009).

A parturiente Número Três descreveu a melhora durante os dois tipos de métodos, porém, o que mais causou impacto no alívio da dor, foram os combinados, a posição preferida por ela foi a sentada na bola suíça, pois segundo ela, proporcionava maior relaxamento. Os benefícios relacionados ao uso da bola suíça estão relacionados ao fato de que assumindo a posição vertical, o eixo axial fetal fica alinhado com o materno e isso facilita a ampliação do ângulo da passagem pélvica e causa relaxamento por meio da movimentação da musculatura.

Na cidade de Santa Maria, mais precisamente na Maternidade do Hospital Casa de Saúde, foi realizado um estudo experimental com um grupo de 10 gestante em trabalho de parto, no qual foi dividido em experimental e de controle. Nesse estudo foi utilizado a bola suíça como método de alívio da dor e por meio dele pode se ter conhecimento sobre a eficácia da utilização da bola no alívio da dor durante o TP (Braz et al., 2014).

Outro estudo que contribui para fortalecer a afirmativa de que a bola suíça pode ser eficaz na diminuição da sensação dolorosa é de um ensaio clinico randomizado e controlado no qual foi utilizado o banho quente e a bola suíça para ser avaliado 
sua influência na progressão do trabalho de parto e ao fim do estudo, concluiu-se que com o uso do banho e da bola associados, além de modificar a progressão do parto, causou alívio da dor. Já o banho não auxiliou na progressão, porém, alíviou significativamente a dor das parturientes (Henrique et al., 2016).

Esses métodos não farmacológicos são estratégias utilizadas no trabalho de parto para aumentar a tolerância da mulher a dor, possibilitando benefícios para a maior parte das mulheres que fazem seu uso. Através dessas técnicas é possível ainda proporcionar diminuição do estresse, ansiedade e tensão, distraindo a atenção do foco doloroso. Estimulam o protagonismo e autonomia da mulher, para escolher a melhor forma de parir e o método que melhor supre sua necessidade de alívio e conforto (Almeida, Acosta \& Pinhal, 2015).

\section{Conclusão}

O resultado da análise dos MNFDP por meio da EVA foi satisfatório para a paciente três, tanto nos métodos combinados como no isolado. Na paciente um, houve satisfatoriedade apenas nos métodos combinados e na paciente dois houve discreta promoção de alívio na utilização do método isolado.

O trabalho de parto como descrito anteriormente é um evento marcante na vida da mulher, seja ele de forma positiva ou negativa e a forma como ele é conduzido será o diferencial entre essas duas vertentes. O resultado desse estudo pôde demostrar que há eficácia dentre os métodos que foram utilizados dentro do contexto das parturientes que foram atendidas, porém, há quesitos determinantes para que esses possam funcionar de forma individual em cada mulher. Deve ser levado em consideração a subjetividade, as escolhas, o conhecimento prévio da parturiente sobre esses métodos, a interação pacienteprofissional, entre outros aspectos.

O alívio da dor não vai garantir à mulher uma experiência menos traumatizante se não houver um acolhimento, conforto e segurança por parte do profissional que irá recebê-la, pois antes de tudo é necessário que haja humanização e respeito. Esses métodos são simples de serem utilizados, de baixo custo e podem ser opção ao uso indiscriminado de medicações. Podem ser instrumentos da autonomia, liberdade de escolha e protagonismo da mulher durante o seu processo parturitivo.

Para que haja maior conhecimento sobre a temática e certeza na eficácia do uso dos MNFDP são necessários mais estudos que contemplem na prática sua avaliação, bem como a opinião daquelas que estão recebendo esses métodos para que se possa compreender a dimensão da dor e as suas melhores formas de alívio.

\section{Referências}

Almeida, A. M. A., \& Souza, M. R. (2012). Sentidos da dor do parto normal na perspectiva e vivência de um grupo de mulheres usuárias do sistema único de saúde. Rev. Min. Enferm, 16(2), 241-250. http://reme.org.br/exportar-pdf/525/v16n2a13.pdf.

Almeida, J. M., Acosta, L. G., \& Pinhal, M. G. (2015). Conhecimento das puérperas com relação aos métodos não farmacológicos de alívio da dor do parto. Rev. Min. Enferm., 19(3), 711-717. http://www.dx.doi.org/10.5935/1415-2762.20150054

Apolinário, D., Rabelo, M., Wolff, L. D. G., Souza, S. R. R. K., \& Leal, G. C. (2016) Práticas na atenção ao parto e nascimento sob a perspectiva das puérperas. Rev Rene, 17(1), 20-28. https://www.redalyc.org/pdf/3240/324044160004.pdf.

Araújo, A., Correia, A., Rodrigues, D., Lima, L., Gonçalves, S., \& Viana, A. (2018). Métodos não farmacológicos no parto domiciliar. Revista de Enfermagem UFPE on line, 12(4), 1091-1096. doi:https://doi.org/10.5205/1981-8963-v12i4a230120p1091-1096-2018

Barbieri, M., Henrique, A. J., Chors, F. M., Maia, N.de L., \& Gabrielloni, Maria Cristina. (2013). Banho quente de aspersão, exercícios perineais com bola suíça e dor no trabalho de parto. Acta Paulista de Enfermagem, 26(5), 478-484. https://doi.org/10.1590/S0103-21002013000500012

Brandolfi, J. A., Minetto, A. I., Pacheco, R., Duminelle, K. G., \& Robsin, E. S. (2017). Atuação fisioterapêutica para redução do quadro álgico no trabalho de parto ativo. Rev. Inova Saúde, 6(2), 20-34. http://dx.doi.org/10.18616/is.v6i2.2554

Brasil. (2012). Ministério da Saúde. Conselho Nacional De Saúde; Portaria $\mathrm{n}^{\circ} 1.459$, de 24 de junho de 2011 . Diário Oficial da União, 2012http://bvsms.saude.gov.br/bvs/saudelegis/gm/2011/prt1459_24_06_2011.html. 
Braz, M., da Rosa, J., Maciel, S., \& Pivetta, H. (2014). Bola do nascimento: recurso fisioterapêutico no trabalho de parto. Cinergis, 15(4). doi:https://doi.org/10.17058/cinergis.v15i4.5199

Castro, S. C., Castro, A. C., \& Mendonça, A. C. (2012). Abordagem fisioterapêutica no pré-parto: proposta de protocolo e avaliação da dor. Fisioterapia e Pesquisa, 19(3), 210-214. https://doi.org/10.1590/S1809-29502012000300004

Davim, R. M. B., Torres, G. V., \& Dantas, J. C. (2009). Efetividade de estratégias não farmacológicas no alívio da dor de parturientes no trabalho de parto. Revista da Escola de Enfermagem da USP, 43(2), 438-445. https://doi.org/10.1590/S0080-62342009000200025

Gallo, R. B. S., Santana, L. S., Marcolin, A. C., Ferreira, C. H. J., Duarte, G. \& Quintana, S. M. (2011). Recursos não-farmacológicos no trabalho de parto: protocolo assistencial. FEMINA, 39(1), 42-45. http://pesquisa.bvsalud.org/portal/resource/pt/lil-594050.

Henrique, A. J., Gabrielloni, M. C., Cavalcanti, A. C. V. Melo, P. S., \& Barbieri, Márcia. (2016). Hidroterapia e bola suíça no trabalho de parto: ensaio clínico randomizado. Acta Paulista de Enfermagem, 29(6), 686-692. https://doi.org/10.1590/1982-0194201600096.

IBGE - Instituto Brasileiro de Geografia e Estatística. (2018). Censo demográfico. https://www.ibge.gov.br/estatisticas-novoportal/por-cidade-estadoestatisticas.html?t=destaques\&amp;c=2208403\&gt.

Katzer, T. (2016). Métodos não farmacológicos para o alívio da dor: percepções da equipe multiprofissional no trabalho de parto e parto (Trabalho de Conclusão de Curso) Repositório UNISC. Santa Cruz do Sul. https://repositorio.unisc.br/jspui/bitstream/11624/1371/1/Ta\% C3\% ADs\%20Katzer.pdf

Carvalho, J. B. L., Texeira, G. A., Lopes, T. R. G. (2015). Humanização do trabalho de parto e nascimento: aplicação de estratégias não farmacológicas efetivas nesse processo. Rev. Enferm. UFPE (online), 9(7), 9133-9138, http://dx.doi.org/10.5205/reuol.8074-70954-1-SM0907supl201525

Nielsen, E., Sabatino, H., \& Lopes, M. H. B. M. (2011). Dor e comportamento de mulheres durante o trabalho de parto e parto em diferentes posições. Rev. Esc. Enferm. USP, Campinas, 45(3), 557-65. www.ee.usp.br/reeusp/.

Oliveira, L. M. N., \& Cruz, A. G. C. (2014). A utilização da bola suíça na promoção do parto humanizado. Rev. Brasileira de Ciências da Saúde, 18(2), 175180, http://periodicos.ufpb.br/index.php/rbcs/article/viewFile/16698/12924.

Oliveira, M. A. P., Velarde, G. C., \& DE SÁ, R. A. (2015). Entendendo a pesquisa clínica V:Relatos e séries de casos. FEMINA. 43(5), 236-238. http://files.bvs.br/upload/S/0100-7254/2015/v43n5/a5320.pdf.

Organização mundial da saúde. (1996). Assistência ao parto normal: um guia prático.

Osório, S. M. B., Junior, L. G. S., \& Nicolau, A. I. O. (2014). Avaliação da efetividade de métodos não farmacológicos no alívio da dor no parto. Rev Rene, 15(1), 174-184. http://repositorio.ufc.br/bitstream/riufc/11708/1/2014_art_smbosorio.pdf.

Patah, L. E. M., \& Malik, A. M. (2011). Modelos de assistência ao parto e taxa de cesárea em diferentes países. Revista de Saúde Pública,45(1), 185194. https://doi.org/10.1590/S0034-89102011000100021

Ritter, K. M. (2012). Manejo não farmacológico da dor em mulheres durante o trabalho de parto em um hospital escola (Trabalho de Conclusão de Curso). LUME, https://lume.ufrgs.br/handle/10183/69750.

Santos, H. F.L., \& Araujo, M. M. (2016). Políticas de humanização ao pré-natal e parto: uma revisão de literatura. Rev. Científica FacMais, 6(2), 55-64, http://revistacientifica.facmais.com.br/wp-content/uploads/2016/07/Artigo-6-POL\%C3\%8DTICAS-DE-

HUMANIZA\%C3\%87\%C3\%83O-AO-PR\%C3\%89-NATAL-E-PARTO.pdf.

Silva, D., Ramos, M., Jordão, V., Silva, R., Carvalho, J., \& Costa, M. (2013). Uso de métodos não farmacológicos para o alívio da dor durante o trabalho de parto normal: revisão integrativa. Revista de Enfermagem UFPE on line, 7(5), 1539-1548. https://doi.org/10.5205/1981-8963-v7i5a11645p4161-4170-2013 